FERMILAB-Conf-96/380

\title{
Particle Dynamics Inside a Quasi-Isochronous Storage Ring
}

\author{
K.Y. Ng et al. \\ Fermi National Accelerator Laboratory \\ P.O. Box 500, Batavia, Illinois 60510
}

October 1996

Submitted to the Proceedings of the XII Advanced ICFA Workshop on Nonlinear and Collective Phenomena in Beam Physics: Theory and Experiments, Arcidosso, Italy, September 2-9, 1996. 


\section{Disclaimer}

This report was prepared as an account of work sponsored by an agency of the United States Government. Neither the United States Government nor any agency thereof, nor any of their employees, makes any warranty, express or implied, or assumes any legal liability or responsibility for the accuracy, completeness or usefulness of any information, apparatus, product or process disclosed, or represents that its use would not infringe privately owned rights. Reference herein to any specific commercial product, process or service by trade name, trademark, manufacturer or otherwise, does not necessarily constitute or imply its endorsement, recommendation or favoring by the United States Government or any agency thereof. The views and opinions of authors expressed herein do not necessarily state or reflect those of the United States Government or any agency thereof.

\section{Distribution}

Approved for public release: further dissemination unlimited. 


\title{
Particle Dynamics Inside a Quasi-Isochronous Storage Ring
}

\author{
K.Y. Ng \\ Fermi National Accelerator Laboratory, ${ }^{1}$ P.O. Box 500, Batavia, IL 60.510 \\ M. Bai, B. Brabson, C.M. Chu, X. Kang, \\ D. Jeon, S.Y. Lee, A. Riabko, X. Zhao \\ Department of Physics, Indiana University, Bloomington, IN 47405
}

\begin{abstract}
Quasi-isochronous $\alpha$-like bucket can be important in providing ultra short bunches. The instability of a bunch inside the bucket is found to be severely affected by rf phase modulation. The synchrotron tune drops to zero at the bucket edge very rapidly, indicating the possibility of a thick chaotic layer due to overlapping of resonance islands. The approach to chaos comes from a sequence of bifurcation into 2:1 parametric resonances. When quantum excitation is included in addition to radiation damping, the instability is worsened. The steady-state particle distribution in the longitudinal phase space is Gaussian in the phase coordinate and non-Gaussian in the momentum coordinate, unless the bunch is small. The size of the bunch is governed only by the "thermal Energy" $E_{t h}=$ $D^{2} / 2 A$, where $D$ and $A$ are, respectively, the normalized diffusion and damping coefficients. The quantum lifetime of the particle bunch, for the $D$ and $A$ that have been enhanced by the smallness of the phase-slip factor, is studied and turns out to be much longer than expected. Phase modulation tends to enhance quantum diffusion at high frequencies, but leads to stochastic resonances instead at low frequencies.
\end{abstract}

\section{INTRODUCTION}

Sub-millimeter electron bunches can be important in time-resolved experiments, next generation light sources, coherent synchrotron radiation, and damping rings in future linear colliders. One method to achieve such small bunch length is to lower the phase-slip factor $\eta$, which is defined as

$$
\frac{\Delta T}{T_{0}}=\eta \delta
$$

where $T_{0}$ is the revolution period of the synchronous particle around the storage ring and $\Delta T$ is the increase in period for a particle with a fractional

1) Operated by the Universities Research Association, Inc., under contract with the U.S. Department of Energy.

(C) 1995 American Institute of Physics 
momentum offset $\delta$. The phase-slip factor is momentum dependent and can be expanded as

$$
\eta=\eta_{0}+\eta_{1} \delta+\eta_{2} \delta^{2}+\cdots
$$

When $\left|\eta_{0}\right|$ is reduced to a small enough value, the first order term $\eta_{1} \delta$ becomes important. The Hamiltonian governing the motion of a beam particle is therefore

$$
H=\frac{1}{2} h \eta_{0} \delta^{2}+\frac{1}{3} h \eta_{1} \delta^{3}+\frac{e V_{\mathrm{rf}}}{2 \pi \beta^{2} E_{0}}\left[\cos \left(\phi_{s}+\Delta \phi\right)+\Delta \phi \sin \phi_{s}\right],
$$

where $\Delta \phi$, canonical to $\delta$, is the rf phase offset from the synchronous phase $\phi_{s}, V_{\mathrm{rf}}$ is the rf voltage, $E_{0}$ is the energy of the synchronous particle and $\beta$ its velocity with respect to the velocity of light. At large $\left|\eta_{0} / \eta_{1}\right|$, the Hamiltonian represents two series of pendulum-like buckets. As $\left|\eta_{0} / \eta_{1}\right|$ decreases to a point when the values of the Hamiltonian through all unstable fixed points are equal, the two series merge. When [1]

$$
\left|\frac{\eta_{0}}{\eta_{1}}\right|<\left\{\frac{6 e V_{\mathrm{rf}}}{\pi \beta^{2} h \eta_{0} E}\left[\left(\frac{\pi}{2}-\phi_{s}\right) \sin \phi_{s}-\cos \phi_{s}\right]\right\}^{1 / 2}
$$

the buckets becomes $\alpha$-like and the storage ring is said to be quasi-isochronous (QI). By the way, the right-hand side of Eq. (1.4) is just $\sqrt{3}$ times the half bucket height when the $\eta_{1}$ term in the Hamiltonian is absent. By the deployment of sextupoles, the contribution of $\eta_{1}$ can be eliminated, and the next order $\eta_{2}$ will restore the bucket to pendulum-like, even if the zeroth order $\eta_{0}$ vanishes. However, the $\alpha$-like QI bucket has its own merit of being much narrower than the pendulum-like bucket. Therefore, if one needs an ultra short bunch, such a bucket may become indispensable [1-8].

The kinematics of the QI bucket are reviewed in Sec. II. Phase modulation is studied in Sec. III when radiation damping is included. The critical modulation amplitude for the stable 1:1 parametric resonance is computed and also the threshold of bifurcation into the $2: 1$ parametric resonance. It is then demonstrated that global chaos is the result of a series of bifurcation into 2:1 parametric resonances. Quantum excitation is included in Sec. IV. The steady-state particle distribution inside a bunch is found to follow the contours of the non-perturbative Hamiltonian that does not contain damping and excitation. When the bunch is small, it is bi-Gaussian in shape with the rms spreads governed by the "thermal energy" $E_{t h}=D^{2} / 2 A$, where $D$ and $A$ are the normalized diffusion and damping coefficients. The quantum lifetime is next studied. Although $D$ and $A$ for the QI system have been heavily enhanced by the vanishingly small phase-slip factor, the quantum lifetime turns out to be very much longer than expected. When phase modulation is introduced, we find that the situation of global chaos becomes worsened. 
For small modulation amplitudes, the steady-state distribution remains biGaussian and is still determined by the same "thermal energy" $E_{t h}$. At high modulation frequencies, quantum diffusion is enhanced and the lifetime shortened. However, at low modulation frequencies, stochastic resonances develop. Touschek scattering is discussed in Sec. V, where we find that beam loss will be enhanced because of the smaller momentum aperture in the QI bucket. Finally, conclusions are given in Sec. VI.

Most of the material in this presentation is taken from three previous articles $[9-11]$.

\section{KINEMATICS}

The independent "time" variable in the Hamiltonian of Eq. (1.1) is $\theta$ which increases by $2 \pi$ in one turn. Now we use $t=\nu_{s} \theta$ as the "time variable", which increases by $2 \pi$ during one synchrotron period $T_{0} / \nu_{s}$, where

$$
\nu_{s}=\sqrt{\frac{h e V_{\mathrm{rf}}\left|\eta_{0} \cos \phi_{s}\right|}{2 \pi \beta^{2} E_{0}}}
$$

is the synchrotron tune at small amplitude. Changing to the new canonical variables,

$$
x=-\frac{\eta_{1}}{\eta_{0}} \delta, \quad p=\frac{d x}{d t},
$$

the Hamiltonian is transformed to a universal form

$$
H=\frac{p^{2}}{2}+\frac{x^{2}}{2}-\frac{x^{3}}{3} .
$$

The "energy" $E$, or the value of the Hamiltonian, is a constant of motion and varies between 0 and $\frac{1}{6}$ from the center to the edge of the QI bucket. We have made the assumption that $\Delta \phi$ is small when Eq. (2.3) is derived. However, a $\alpha$-like QI bucket is always narrow in rf phase extent. Therefore Eq. (2.3) does represent an excellent approximation to the Hamiltonian for such a bucket for any synchronous phase $\phi_{s}$.

At fixed "energy" $E$, the equation of motion is

$$
\left(\frac{d x}{d t}\right)^{2}=\frac{2 x^{3}}{3}-x^{2}+2 E .
$$

which becomes the Weierstrass equation $[12,13]$, If we further make the substitutions $t=\sqrt{6} u$ and $x=\wp$ :

$$
\left(\frac{d \wp}{d u}\right)^{2}=4\left(\wp-e_{1}\right)\left(\wp-e_{2}\right)\left(\wp-\epsilon_{3}\right) .
$$


The 3 turning points are, respectively,

$$
\begin{aligned}
& e_{1}=\frac{1}{2}+\cos \xi \\
& e_{2}=\frac{1}{2}+\cos \left(\xi-\frac{2 \pi}{3}\right), \\
& \epsilon_{3}=\frac{1}{2}+\cos \left(\xi+\frac{2 \pi}{3}\right),
\end{aligned}
$$

with

$$
\xi=\frac{1}{3} \cos ^{-1}(1-12 E)
$$

For particles inside the separatrix or bucket, the discriminant $\Delta=648 E(1-$ $6 E)$ is positive, and the Weierstrass $\wp$ function can be expressed in terms of the Jacobian elliptic function. We have therefore

$$
\begin{aligned}
& x(t)=\epsilon_{3}+\left(\epsilon_{2}-\epsilon_{3}\right) \operatorname{sn}^{2}\left(\sqrt{\frac{e_{1}-e_{3}}{6}} t \mid m\right), \\
& m=\frac{e_{2}-\epsilon_{3}}{e_{1}-\epsilon_{3}}=\frac{\sin \xi}{\sin \left(\xi+\frac{\pi}{3}\right)} .
\end{aligned}
$$

It is worth pointing out that, contrary to the usual custom, the variable $x$ has been used to denote momentum spread while the canonical conjugate $p$ has been used to denote phase. This notation is used because we can talk about a "potential well" of $U(x)=\frac{1}{2} x^{2}-\frac{1}{3} x^{3}$ in this way, and Eq. (2.4) or Eq. (2.5) just describes the motion of a particle in the potential well. This potential well has a barrier separating the stable bucket from the unstable part of the phase space. This barrier is located at the unstable fixed point (UFP) through which the separatrix passes through. The separatrix orbit, which corresponds to $m=1$. is given by

$$
x_{s x}(t)=1-\frac{3}{\cosh t+1}, \quad p_{s x}(t)=\frac{3 \sinh t}{(\cosh t+1)^{2}} .
$$

The action $J$ can be readily derived,

$$
J=\frac{1}{2 \pi} \oint p d x=\frac{1}{8} \sqrt{\frac{2}{3}}\left(\epsilon_{2}-\epsilon_{3}\right)^{2} \cdot\left(\epsilon_{1}-\epsilon_{3}\right)^{1 / 2} F\left(\frac{3}{2},-\frac{1}{2} ; 3 ; m\right),
$$

where $F$ is the hypergeometric function $[12,13]$, and so is the synchrotron tune,

$$
\nu_{s} Q(E)=\nu_{s} \frac{d E}{d J}=\nu_{s}\left[\frac{\pi \sin ^{\frac{1}{2}}\left(\xi+\frac{\pi}{3}\right)}{2^{\frac{1}{2}} 3^{\frac{1}{4}} K(m)}\right]
$$

where $K(m)$ is the complete elliptical function of the first kind. This synchrotron tune is shown in Fig. 1 as a function of the energy $E$ along with the 


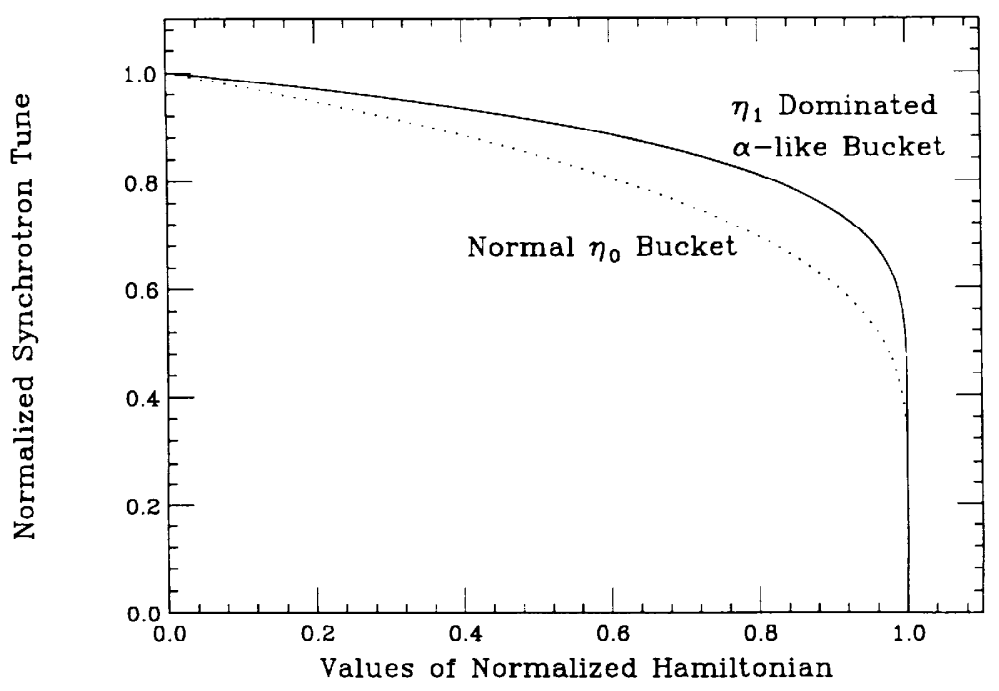

FIGURE 1. Normalized synchrotron tune inside the $\alpha$-like bucket as a function of amplitude of oscillation. Note that the drop is very abrupt at the bucket edge corresponding to a normalized Hamiltonian of value $H=1$. For comparison, the normalized synchrotron tune inside the usual pendulum-like bucket is shown in dots.

synchrotron tune for the usual pendulum bucket. We note particularly that the synchrotron tune decreases to zero very sharply near the separatrix. Because of the sharp decrease in synchrotron tune, time dependent perturbation will cause overlapping parametric resonances and chaos near the separatrix [14-16].

The conjugate angle variable is

$$
\psi=\frac{\partial F_{2}}{\partial J}=Q t
$$

which is obtained from the generating function

$$
F_{2}(x, J)=\int_{\epsilon_{3}}^{x} p d x .
$$

\section{PHASE MODULATION}

In the presence of rf phase noise of tune $\nu_{m}$, the Hamiltonian becomes time dependent,

$$
H=\frac{p^{2}}{2}+\frac{x^{2}}{2}-\frac{x^{3}}{3}+\omega_{m} B x \cos \omega_{m} t .
$$

where $\omega_{m}=\nu_{m} / \nu_{s}$ is the normalized modulation tune, and $B$, the normalized modulation amplitude, is related to the $\mathrm{rf}$ phase modulation $a$ in the original $(\Delta \phi, \delta)$ coordinates by 

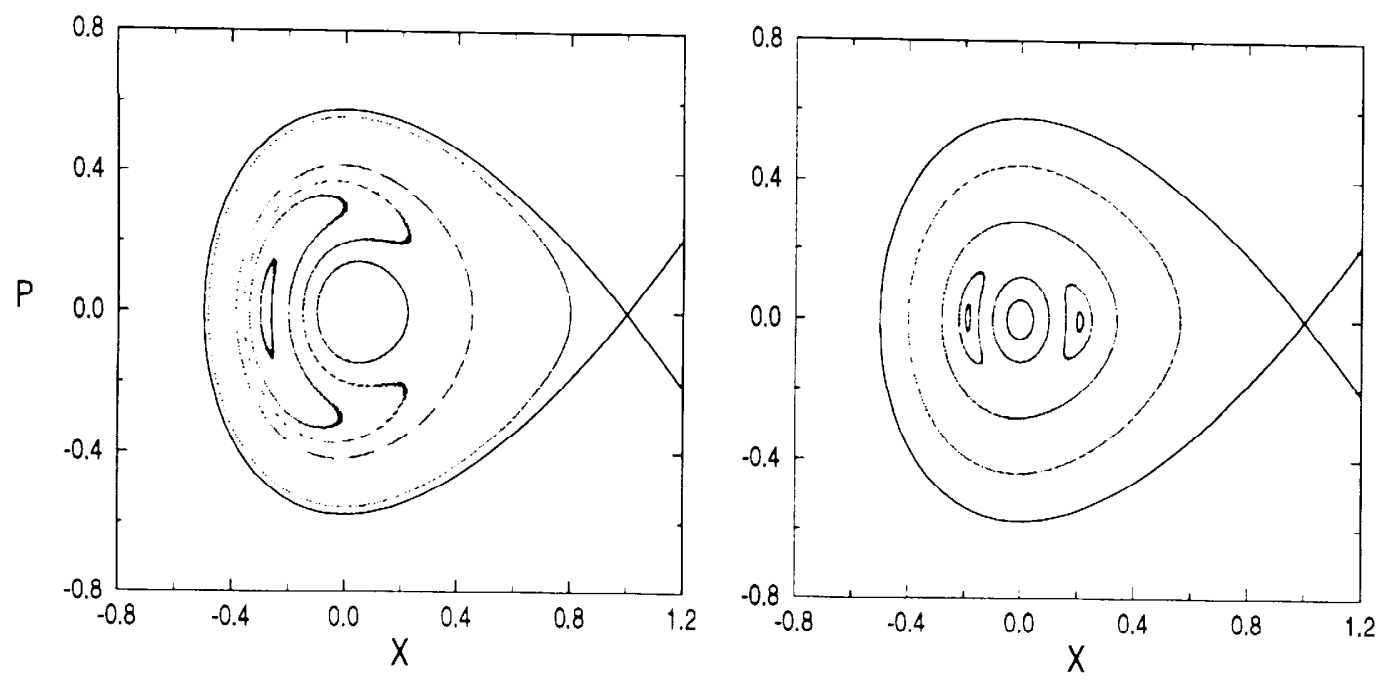

FIGURE 2. Poincaré surfaces of section for a QI Hamiltonian with $B=0.003$ and $\omega_{m}=0.96$ (left) and $B=0.0055$ and $\omega_{m}=1.97$ (right). Here $B$ and $\omega_{m}$ are rf phase modulation amplitudes and tunes respectively. The separatrix trajectory is shown in this case for marking the boundary of stable motion of the unperturbed Hamiltonian. In reality, the separatrix is destroyed by the harmonic modulation.

$$
B=\frac{\eta_{1} a}{\eta_{0} \nu_{s}}
$$

As a result, the modulation is very much enhanced for the QI storage ring due to the vanishingly small value of $\eta_{0}$. The perturbed part of the Hamiltonian can be easily expanded as a Fourier series in the angle variable $\psi$ :

$x \cos \omega_{m} t=\sum_{n=0}^{\infty} g_{n} \cos n \psi \cos \omega_{m} t=\frac{1}{2} \sum_{n=0}^{\infty} g_{n}\left[\cos \left(n \psi-\omega_{m} t\right)+\cos \left(n \psi+\omega_{m} t\right)\right]$.

which exhibits the $n: 1$ resonance when the normalized modulation tune $\omega_{m}$ is close to the integer $n$. Here, the modulation strength $g_{n}$ for $n \neq 0$ is given by

$$
g_{n}(J)=\frac{1}{\pi} \int x \cos n v d \psi=\frac{2 \pi^{2}\left(e_{1}-e_{3}\right)}{K^{2}(m)} \frac{(-1)^{n} n q^{n}}{1-q^{2 n}},
$$

with $q=\exp \left[-\pi K^{\prime}(m) / K(m)\right]$. For the usual pendulum-like bucket, because of the symmetry between positive and negative momentum spread, only resonances corresponding to odd $n$ occur. Here, such a symmetry is absent, we have resonances corresponding to both odd and even $n$. For example, Fig. 2 shows separately Poincaré surfaces for 1:1 and 2:1 resonances, respectively. 


\section{A Radiation Damping}

Electrons emit radiation which leads to synchrotron radiation damping. The equation of motion from the Hamiltonian of Eq. (3.1) will be modified to

$$
x^{\prime \prime}+A x^{\prime}+x-x^{2}=-\omega_{m} B \cos \omega_{m} t,
$$

where the normalized damping coefficient is given by

$$
A=\frac{\lambda}{\nu_{s}}=\frac{U_{o} J_{E}}{2 \pi E_{0} \nu_{s}} .
$$

Here, $\lambda$ is the damping decrement, $U_{0}$ the energy loss per turn, and $J_{E}$ the damping partition number. Note that, due to the small synchrotron tune of the QI system, the normalized damping coefficient is again enhanced. Some typical damping parameters for storage rings are listed in Table 1. Thus, in our study here, it is necessary to allow $A$ to reach $\sim 0.5$.

TABLE 1. Typical parameters of electron storage rings

\begin{tabular}{lrrrrr}
\hline & LEP & HERB & APS & CESR & ALS \\
\hline$E_{0}(\mathrm{GeV})$ & 55 & 9 & 7 & 6 & 1.5 \\
$U_{0} / E_{0}\left(10^{-3}\right)$ & 4.8 & 0.39 & 0.78 & 0.32 & 0.074 \\
$\alpha_{c 0}\left(10^{-4}\right)$ & 3.9 & 24.4 & 2.4 & 152 & 14 \\
$\nu_{s}$ (damping) & 0.085 & 0.05 & 0.0066 & 0.064 & 0.0082 \\
$A$ dam & 0.018 & 0.0024 & 0.037 & 0.002 & 0.003 \\
$f_{0}(\mathrm{kHz})$ & 11.2 & 136 & 283 & 390 & 1524 \\
$f_{\text {syn }}(\mathrm{kHz})$ & 0.956 & 6.8 & 1.9 & 25 & 12.5 \\
\hline
\end{tabular}

\section{B 1:1 Parametric Resonance}

In the presence of damping, the stable fixed points of the Hamiltonian of Eq. (3.1) become attractors, towards which the Poincaré sections spiral. The attractors are periodic solutions of the Equation of motion (3.5). An approximate solution can be obtained using the method of harmonic linearization. For the 1:1 resonance, we use the ansatz

$$
x=X_{0}+X_{1} \cos \left(\omega_{m} t+\chi_{1}\right) .
$$

Substituting into Eq. (3.5) and neglecting all higher harmonics, we obtain

$$
\begin{gathered}
\omega_{m}^{2} B^{2}=A^{2} \omega_{m}^{2} X_{1}^{2}+\left(\omega_{m}^{2}-\sqrt{1-2 X_{1}^{2}}\right)^{2} X_{1}^{2}, \\
X_{0}=\frac{1}{2}\left(1-\sqrt{1-2 X_{1}^{2}}\right)^{2} X_{1}^{2},
\end{gathered}
$$




$$
\chi_{1}=-\frac{\omega_{m} A}{\omega_{m}^{2}-\sqrt{1-2 X_{1}^{2}}} .
$$

It is obvious that a maximum amplitude $X_{1}=2^{-1 / 2}$ exists, which lead to a maximum tolerable modulation amplitude for the 1:1 parametric resonance of

$$
B_{c r, 1: 1}=\left(\frac{A^{2}+\omega_{m}^{2}}{2}\right)^{1 / 2} \approx \frac{\omega_{m}}{\sqrt{2}} \quad \omega_{m} \geq 1 .
$$

The existence of a critical modulation amplitude is a result of the potential well in the Hamiltonian of Eq. (3.1). When the modulation is too strong, particles spill over the potential barrier and get lost.

\section{Bifurcation and chaos}

When the modulation tune is reduced to $\omega_{m} \leq 2$, bifurcation takes place because of the presence of the $2: 1$ parametric resonance. We extend the previous analysis by adding a perturbation $y(t)$ to ansatz in Eq. (3.7):

$$
x=X_{0}+X_{1} \cos \left(\omega_{m} t+\chi_{1}\right)+y(t) \text {. }
$$

Neglecting higher harmonics, we find $y(t)$ satisfies the damped Mathieu equation,

$$
y^{\prime \prime}+A y+\left[1-2 X_{0}-2 X_{1} \cos \left(\omega_{m} t+\chi_{1}\right)\right] y \approx 0
$$

and the solution can be written as

$$
y(t)=X_{1 / 2} \cos \left(\frac{1}{2} \omega_{m} t+\chi_{\frac{1}{2}}\right),
$$

where $X_{1 / 2} \sim e^{s t}$ with $s \leq 0$. Mathieu instability then implies

$$
\left(\frac{1}{2} \omega_{m}^{2}-\sqrt{1-2 X_{1}^{2}}\right)^{2}+\frac{1}{4} A^{2} \omega_{m}^{2} \leq X_{1}^{2}
$$

from which the threshold $X_{1(2: 1)}$ can be solved as a function of $A$ and $\omega_{m}$. Substituting the solution in Eq. (3.8) gives the threshold modulation amplitude $B_{2: 1}=B_{2: 1}\left(X_{1(2: 1)}, A, \omega_{m}\right)$ for bifurcation into the $2: 1$ parametric resonance. This threshold $B_{2: 1}$ is plotted in Fig. 3 versus $\omega_{m}$ for different damping parameters. Also plotted is the critical 1:1 modulation amplitude $B_{c r, 1: 1}$, which meets $B_{2: 1}$ at a cusp. This point corresponds to the bifurcation threshold of the 2:1 parametric resonance on top of the limiting stable orbit of the $1: 1$ parametric resonance. Note that the threshold tune of the $2: 1$ bifurcation is lowered as the damping becomes heavier. 


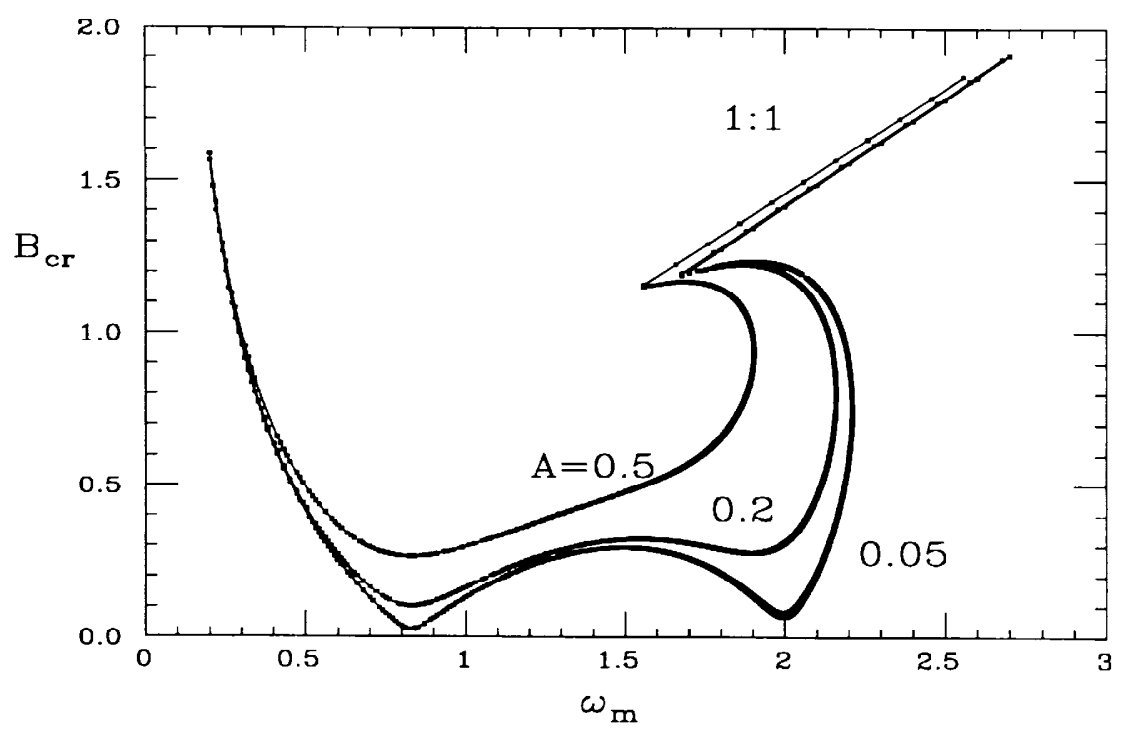

FIGURE 3. The Arabian boot shaped curves correspond to the threshold modulation amplitude $B_{2: 1}$ for the 2:1 parametric resonance with damping parameter $A=0.05,0.2,0.5$ respectively. The critical modulation amplitude $B_{c r, 1: 1}$ for the $1: 1$ parametric resonance is also marked on the figure. The intercept of $B_{2: 1}$ and $B_{c r .1: 1}$, forming a cusp, corresponds to the bifurcation threshold of the $2: 1$ resonance on top of the limiting stable orbit for the 1:1 parametric resonance.

Next tracking is done to locate the critical modulation amplitude $B_{c r}$ so that all the particles inside the bucket are lost or global chaos occurs. This instability boundary is shown in Fig. 4 as thin solid curves in each of the 2 plots.

We note that the $2: 1$ bifurcation threshold is very close to the stability curve in each situation of Fig. 4, indicating that the 2:1 parametric resonance plays an important role in the stability of the QI system. We increase the modulation amplitude $B$ at fixed modulation tune $\omega_{m}=1.975$ for damping parameter $A=0.2$. According to the lower plot of Fig. 4, we should first see a 2:1 bifurcation, then chaotic behavior, and then back to stability. Detail tracking results in Fig. 5 actually show a first bifurcation into 2:1 parametric resonance at $B \sim 0.29$ and another one at $B \sim 0.66$. The sequence of $2: 1$ bifurcations continues until $B \sim 0.73$ when the resonances overlap and chaos results. However, when the modulation amplitude is increased to $B \sim 1.05$, stability is restored through a reversed sequence of period-two bifurcation.

We next study the stability at modulation tune $\omega_{m}=2.0$ and damping parameter $A=0.2$. The first $2: 1$ bifurcation takes place as the modulation amplitude increases to $B \sim 0.30$, as shown in the top plot of Fig. 6 , and the second bifurcation at $B \sim 0.75$. However, as is indicated in the lower plot of Fig. 4, there is no global chaos. We therefore see the attractors recombine in sequence back to the 1:1 resonance. Similar situation applies when the 


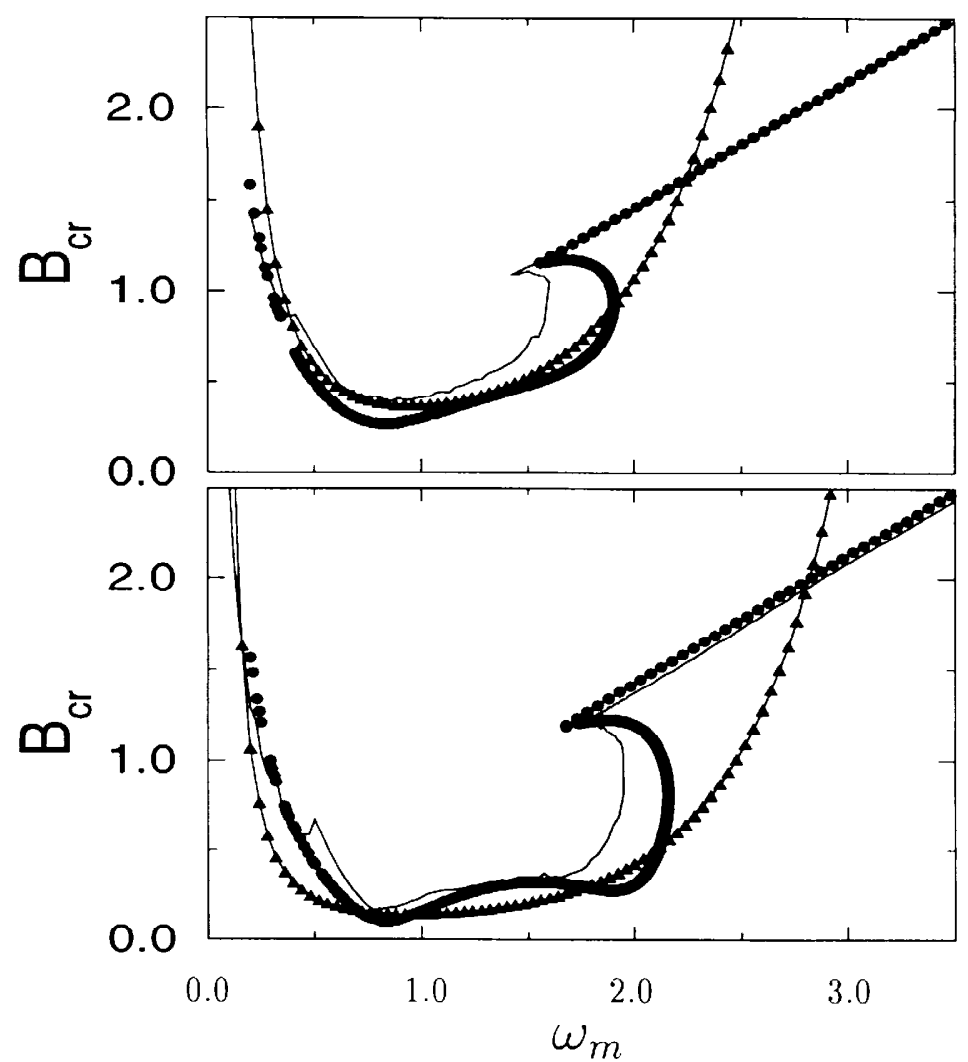

FIGURE 4. The critical phase modulation amplitude $B_{c r}$ (thin solid lines) obtained from numerical simulations is shown as function of $\omega_{m}$ for $A=0.2$ (lower plot) and $A=0.5$ (top plot). The lines joining triangular symbols are $B_{c r}$ obtained from the Melnikov integral method. Circle dots are $B_{c r, 1: 1}$ and $B_{2: 1}$ for the 1:1 and 2:1 parametric resonances. Note that the cusp in $B_{c r}$ observed in numerical simulations is due to the transition from the $2: 1$ to the 1:1 parametric resonances.

modulation harmonic $\omega_{m}=2.1$ as indicated in the lower plot of Fig. 6 .

It is worthwhile to point out that these detailed bifurcations and instability boundaries are not obtained from the usual turn by turn tracking using a small synchrotron tune. Instead. a fourth order symplectic integrator has been used. In other words, this represents the solution of the differential equation (3.5).

To further demonstrate the important role of the $2: 1$ parametric resonance towards global chaos, a study has also been made by fixing the modulation amplitude at $B=0.5$ with damping parameter $A=0.5$ (the top plot of Fig. 4). By decreasing the modulation tune, we see in Fig. 7 the first periodtwo bifurcation at $\omega_{m} \sim 1.52$ and a second one at $\omega_{m} \sim 1.45$ The bifurcation scquence continues until global chaos is attained.

The positions of the attractors are also followed in Fig. 8. At $\omega_{m}=1.540$, there is only one attractor shown as a diamond, which has bifurcated into two 


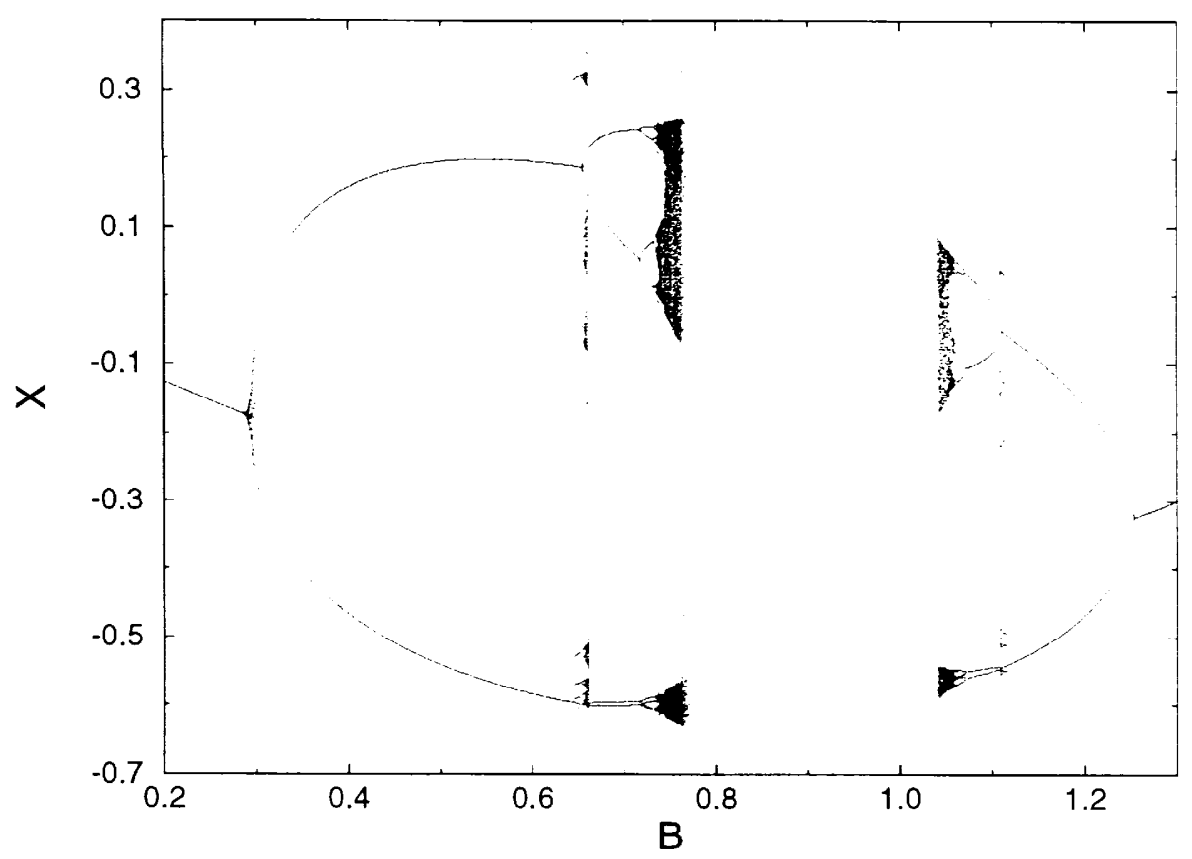

FIGURE 5. The coordinate $x$ of the Poincare surface of section is plotted as a function of the modulation amplitude $B$ at $\omega_{m}=1.975, A=0.2$. A sequence of period-two bifurcations is observed leading to global chaos.

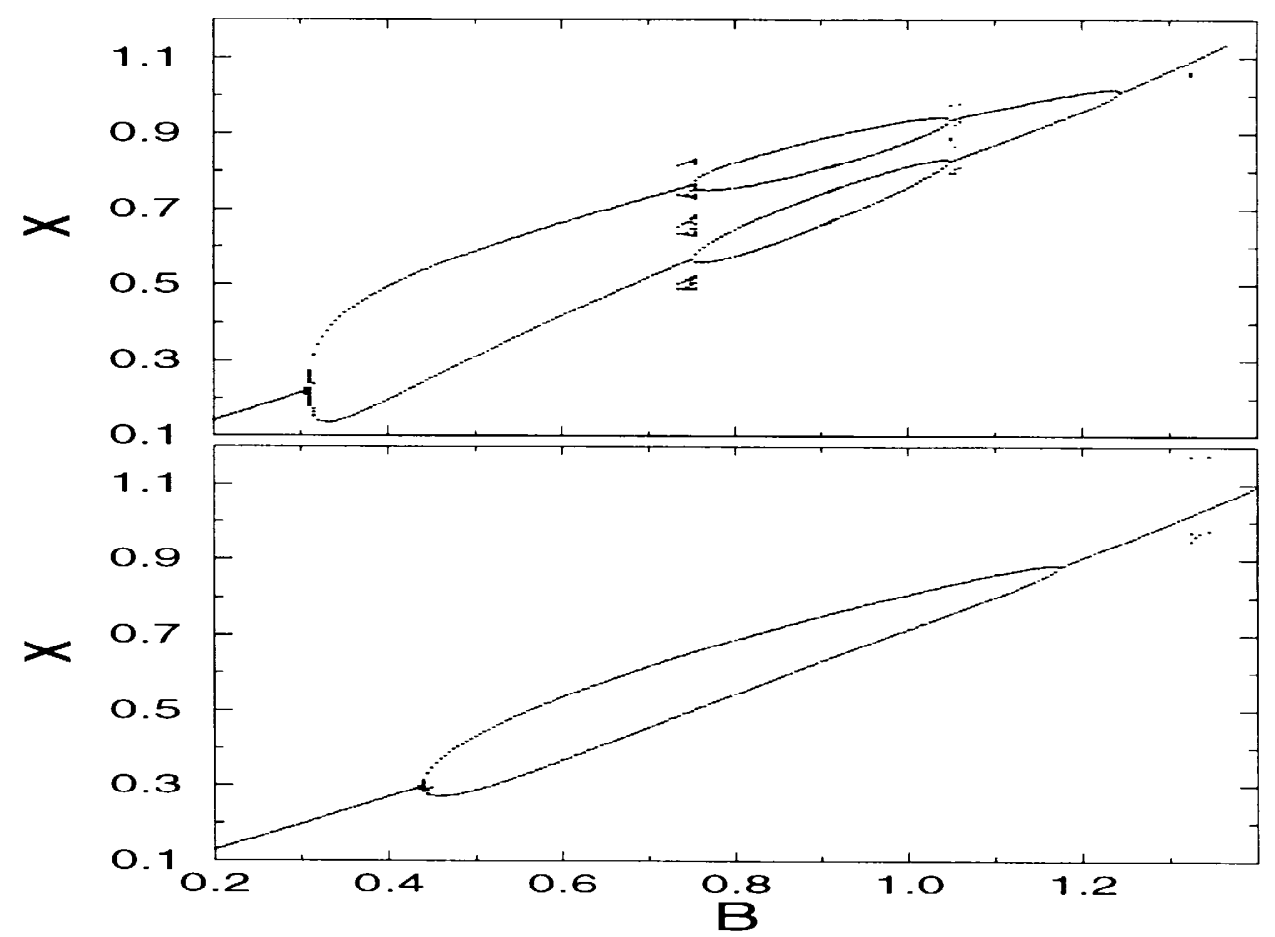

FIGURE 6. The coordinate $x$ of the Poincare surface of section is plotted as a function of the modulation amplitude $B$ at $\omega_{m}=2.0$ (upper plot) and 2.1 (lower plot) with $A=0.2$. 


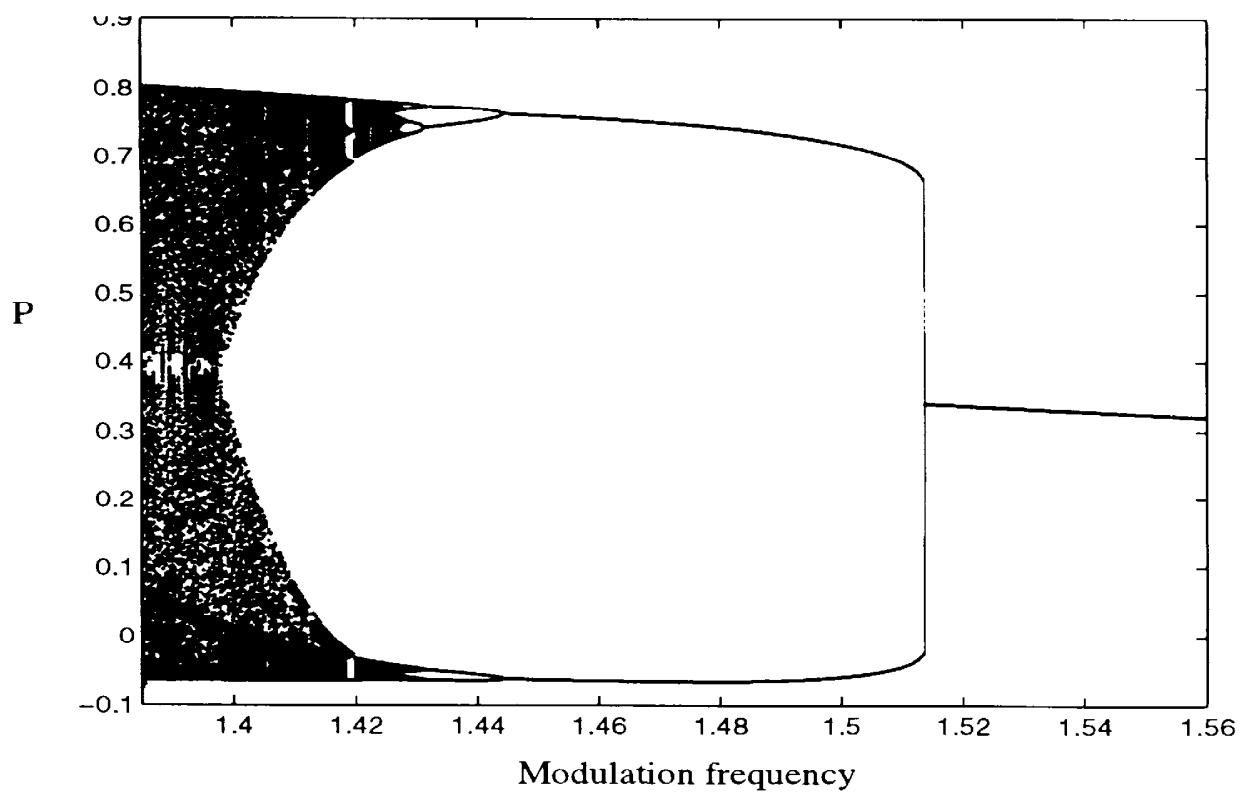

FIGURE 7. Coordinate $p$ of the attractor in the Poincare surface of section is plotted as a function of the modulation frequency (tune) $\omega_{m}$ near the region of global chaos for parameters $A=0.5$ and $B=0.5$. the attractor bifurcates into two at the modulation tune of about 1.515 , then follows a series of period-two bifurcations before reaching global chaos.

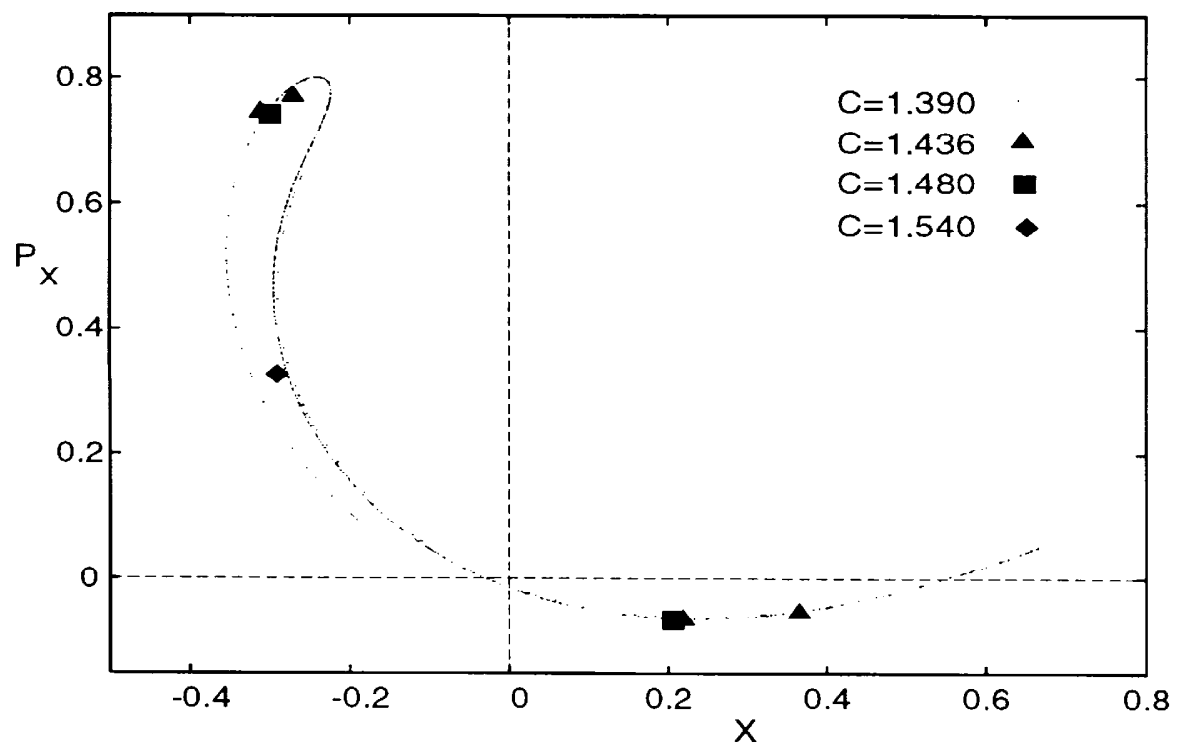

FIGURE 8. In the phase space, a single attractor (diamond) with $A=0.5, B=0.5$ at $\omega_{m}=1.54$ associated with the $1: 1$ parametric resonance bifurcates into 2 attractors (rectangles) related to the 2:1 parametric resonance. Attractors (triangles) are unambiguously identified as the second period-two bifurcation into the secondary (2:1) parametric resonances within the primary (2:1) resonance islands. Dots correspond to the strange attractor with global chaos at $\omega_{m}=1.39$. 
(rectangles) at $\omega_{m}=1.480$, and into four (triangles) at $\omega_{m}=1.436$ The system has become globally chaotic when $\omega_{m}=1.390$, where many bifurcations have taken place. There are many attractors (dots) located along a curve but close to each other. In the turn-by-turn Poincaré map, we see a particle jump from one attractor to the other ramdonly. If there were no damping, we would expect the particle jumping over everywhere in the Poicaré map. This ramdon jumping among the attractors is also demonstrated by the Fourier transform of the steady-state solution, which is plotted in Fig. 9. At $\omega_{m}=1.390$, besides the line of the modulation tune, we see a noise-like structure.

It is interesting to see that some of the bifurcated attractors in Figs. 7 and 8 fall outside the QI bucket with $|p|>1 / \sqrt{3}$. This is because damping has been included, which makes the basin of stability larger than the QI bucket.

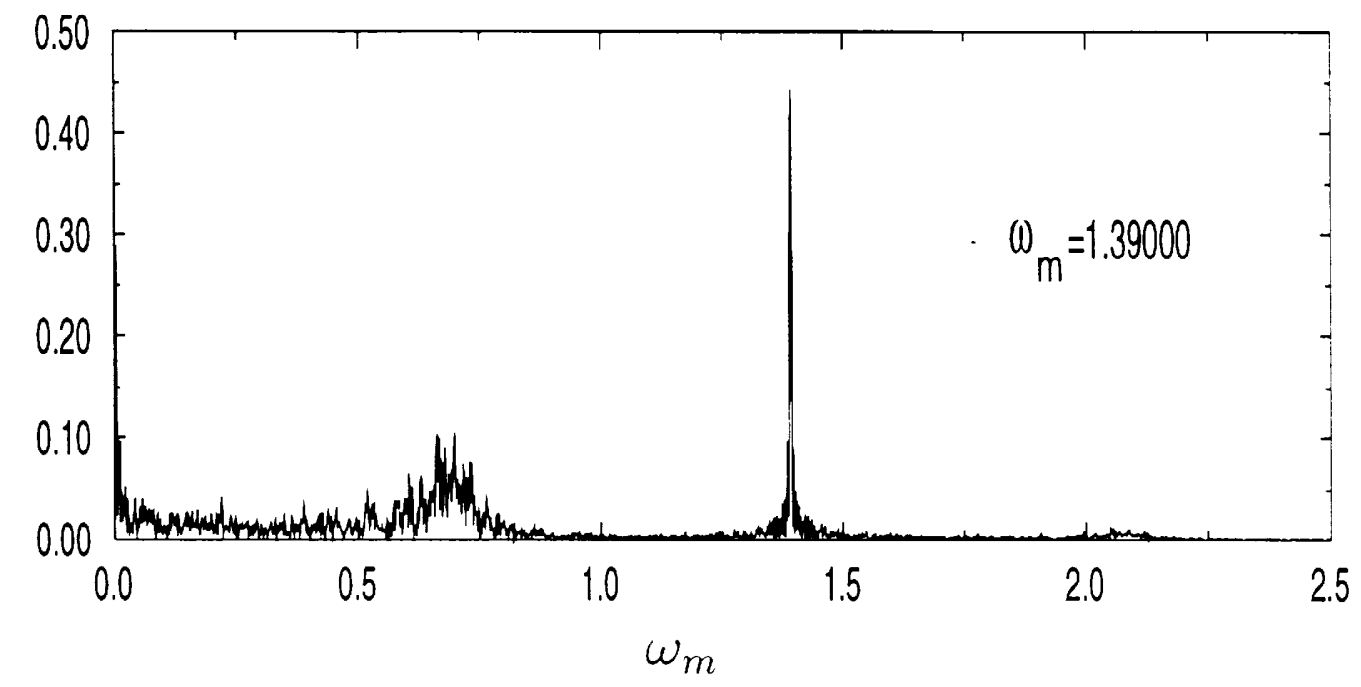

FIGURE 9. FFT spectrum of steady state solution with parameters $A=0.5, B=0.5$, and $\omega_{m}=1.39$ is plotted.

\section{STOCHASTIC BEAM DYNAMICS}

Electrons emit photons randomly, so there are quantum excitations. Equation of motion in Eq. (3.5) becomes

$$
\frac{d^{2} x}{d x 2}+A \frac{d x}{d t}+\frac{d U}{d x}=-\omega_{m} B \cos \left(\omega_{m} t+\phi\right)+D \xi(t)
$$

where $\xi(t)$ is a Langevin force which has the properties that

$$
\langle\xi(t)\rangle=0, \quad\left\langle\xi(t) \xi\left(t^{\prime}\right)\right\rangle=\delta\left(t-t^{\prime}\right) .
$$

Here $U(x)=\frac{1}{2} x^{2}-\frac{1}{3} x^{3}$ is the same "potential" we used before and $D$ is the diffusion coefficient. 


\section{A EQUILIBRIUM DISTRIBUTION}

With quantum excitations, particles will not be damped to attractors. Instead, they spread around an attractor as a bunch. The distribution $\Psi(p, x)$ in the longitudinal phase space is governed by the Fokker-Planck equation,

$$
\frac{\partial \Psi}{\partial t}=\left[-p \frac{\partial}{\partial x}+A \frac{\partial}{\partial p} p+\left(x-x^{2}\right) \frac{\partial}{\partial p}+\frac{D^{2}}{2} \frac{\partial^{2}}{\partial p^{2}}+B \omega_{m} \sin \left(\omega_{m} t+\phi\right) \frac{\partial}{\partial p}\right] \Psi .
$$

which is associated with the Langevin equation of Eq. (4.1). In the case of zero phase modulation $(B=0)$, the solution of the Fokker-Planck equation gives the normalized steady-state distribution of a bunch containing $N$ paricles,

$$
\frac{d N}{d E}=\frac{N}{E_{t h}} e^{-E / E_{t h}},
$$

where the "energy" $E$ is the value of the unperturbed Hamiltonian, and "thermal" energy $E_{\text {th }}$ is given by the Einstein relation, or the fluctuation-dissipation theorem

$$
E_{\mathrm{th}}=\frac{D^{2}}{2 A}
$$

It is worth noting that the iso-density contour of distribution function follows the equi-energy line of the unperturbed Hamiltonian, in spite of the presence of damping and random excitations. The distribution is therefore Gaussian in phase but is only Gaussian in momentum spread provided that the bunch or $E_{\mathrm{th}}$ is small. Since the rms momentum spread for a small electron bunch is given by

$$
\left(\frac{\sigma_{p}}{p_{0}}\right)^{2}=C_{q} \frac{\gamma^{2}}{J_{E} \rho}
$$

for an isomagnetic storage ring, where $\rho$ is the bending radius, $C_{q}=3.84 \times$ $10^{-13} \mathrm{~m}$, and $J_{E}$ is the damping partition number, the diffusion parameter is given by

$$
D=\sqrt{\frac{2 A C_{q}}{J_{E} \rho}}\left|\frac{\eta_{1}}{\eta_{0}}\right| \gamma
$$

Here, the quantum fluctuation coefficient $D$ of the QI dynamical system is also enhanced by the smallness of $\left|\eta_{0}\right|$.

The rms phase space area $\mathcal{A}$ of the beam distribution is given by

$$
\frac{\mathcal{A}}{\pi}=\sqrt{\operatorname{var}(x) \operatorname{var}(p)-(\operatorname{covar}(x, p))^{2}},
$$


where

$$
\begin{aligned}
& \operatorname{var}(x)=\left\langle(x-\langle x\rangle)^{2}\right\rangle, \\
& \operatorname{var}(p)=\left\langle(p-\langle p\rangle)^{2}\right\rangle, \\
& \operatorname{covar}(x, p)=\langle x p\rangle-\langle x\rangle\langle p\rangle .
\end{aligned}
$$

Here $\langle\cdots\rangle$ denotes an average over the beam distribution. In the small bunch approximation, the $\left\langle x^{3}\right\rangle$ term is small and the rms phase space area is equal to $\pi E_{\mathrm{th}}$. Detailed simulations verify the distribution of Eq. (4.4) and also the rms bunch area.

\section{B Quantum Lifetime}

Particles that go outside the bucket through quantum excitation may become lost. The quantum lifetime can be obtained by using the condition of flux conservation. The flux that enters the contour $E=E_{1}$ at any time $t$ is given by

$$
\left.\frac{d N(t)}{d t}\right|_{E_{1}}=\left.\left.\frac{d N(E, t)}{d E}\right|_{E_{1}} \cdot \frac{d E}{d t}\right|_{E_{1}},
$$

where $d N / d E$ is given by Eq. (4.4), and the damping rate $d E / d t$ can be obtained from integrating Eq. (4.1) with

$$
\left.\frac{d E}{d t}\right|_{E_{1}}=-A\left\langle\left(\frac{d x}{d t}\right)^{2}\right\rangle \approx-A E_{1} .
$$

Here, $\left\langle x^{3}\right\rangle$ is again neglected in obtaining the last approximate identity. Substituting Eqs. (4.10) and (4.4) into Eq. (4.9), the quantum lifetime of the bunch is given by

$$
t_{q}=\frac{E_{\mathrm{th}}}{A E_{1}} e^{E_{1} / E_{\mathrm{th}}},
$$

or

$$
\tau_{q}=\frac{E_{\mathrm{th}}}{2 \pi \nu_{s} A E_{1}} e^{E_{1} / E_{\mathrm{th}}} \quad \text { turns, }
$$

which is inversely proportional to the damping parameter $A$. This formula is commonly used in estimating the quantum lifetime for nominal non QI electron storage rings, where the parameter $A$ is small.

Figure 10 shows the quantum lifetime obtained from numerical simulations versus damping parameter $A$ for a constant $E_{\mathrm{th}}=0.03872$. The solid line in Fig. 10 shows the quantum lifetime calculated from Eq. (4.12) with $E_{1}=\frac{1}{6}$, 
corresponding to the separatrix. This result agrees reasonably well with that obtained from numerical simulations only at the small damping limit of $A \leq$ 0.01 . The quantum lifetime from simulations is considerably larger than the prediction of Eq. (4.12) at the medium damping of $A \geq 0.01$. This can be understood by the fact that the effective bucket area for a moderate damping system is larger than that of a weak damping system.

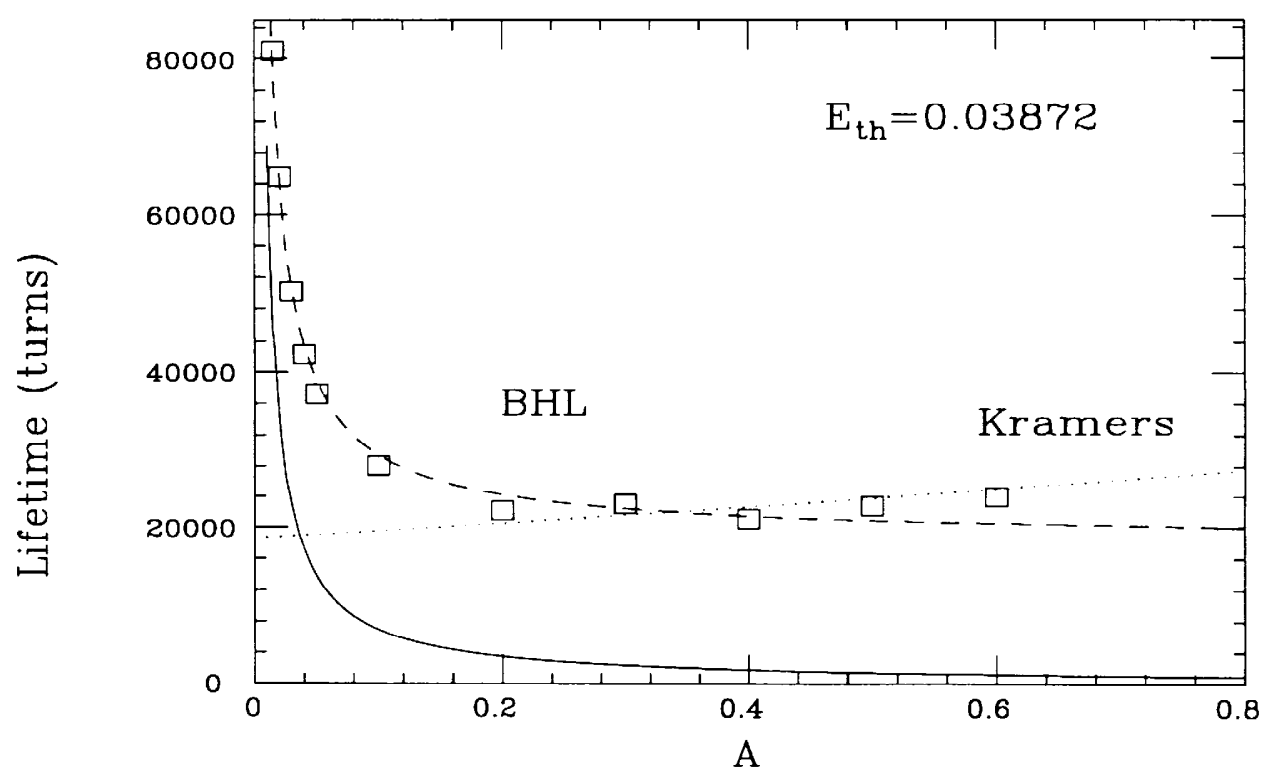

FIGURE 10. The quantum lifetime in number of revolutions obtained from numerical simulations is plotted versus the damping parameter $A$ for a fixed $E_{\mathrm{th}}=0.03872$. Theoretical results are in solid for the weak damping limit, dots for the Kramers's formula, and dashes for the BHL formula.

In the moderate damping regime, there is the Kramers-Chandrasekhar lifetime formula $[17,18]$ shown as dots in Fig. 10,

$$
\tau_{q, \mathrm{KC}}=\left[\left(1+\frac{A^{2}}{4 \omega_{\mathrm{UFP}}^{2}}\right)^{1 / 2}+\frac{A}{2 \omega_{\mathrm{UFP}}}\right] \frac{2 \pi}{\omega_{\mathrm{SFP}}} \epsilon^{E_{1} / E_{\mathrm{th}}},
$$

where $\omega_{\text {SFP }}$ is the natural frequency of the potential at the stable fixed point (SFP), and $\omega_{\text {UFP }}$ is the imaginary frequency at the UFP. There is also the Büttiker-Harris-Landauer (BHL) lifetime [19] formula based on the study of an underdamped Josephson-junction circuit,

$$
\tau_{q, \mathrm{BHL}}=\frac{\left[\left(1+4 E_{\mathrm{th}} / A f E_{1}\right)^{1 / 2}+1\right]^{2}}{4} \frac{2 \pi}{\omega_{\mathrm{SFP}}} \epsilon^{E_{1} / E_{\mathrm{th}}} .
$$

Here $f=7.2$ is the factor relating the separatrix energy to the separatrix action for the QI dynamical system. The BHL formula reduces to Eq. (4.12) in 
the small damping limit with a linear oscillator approximation where $f=2 \pi$. We see that the BHL formula, shown as dashes in Fig. 10, provides a fair agreement with numerical simulations.

\section{STOCHASTICITY AND PHASE MODULATION}

Now let us come back to Eq. (4.1) and study the effect of phase modulation in the presence of stochasticity. Again harmonic linearization is used with the ansatz

$$
x=X_{0}+X_{1} \cos \left(\omega_{m} t+\alpha\right)+u,
$$

where $u$ represents the quantum luctuation and satisfies a Mathieu-Langevin equation,

$$
u^{\prime \prime}+A u^{\prime}+\left[\sqrt{1-2 X_{1}^{2}}-2 X_{1} \cos \left(\omega_{m} t+\alpha\right)\right] u-u^{2}=D \xi(t) .
$$

This provides Mathieu instability to the SFP of the 1:1 parametric resonance, and the instability is the bifurcation into the $2: 1$ parametric resonance. Eventually, global chaos will be reached through a sequence of period-two bifurcations similar to our discussion in Sec. III.

\section{A Equilibrium Distribution}

The Fokker-Planck equation associated with the Mathieu-Langevin equation (5.2) for distribution function $\Psi(u, v, t)$, where $v=u^{\prime}$. is given by

$$
\frac{\partial \Psi}{\partial t}=\left[L_{0}-\frac{\partial}{\partial v}\left(2 X_{1} \cos \left(\omega_{m} t+\alpha\right) u\right)\right] \Psi .
$$

Here $L_{0}$ is the time independent differential operator,

$$
L_{0}=-\frac{\partial}{\partial u} v+\frac{\partial}{\partial v}\left(A v+\sqrt{1-2 X_{1}^{2}} u-u^{2}\right)+\frac{D^{2}}{2} \frac{\partial^{2}}{\partial v^{2}} .
$$

The solution of time independent Fokker-Planck equation is

$$
\Psi_{0}=\frac{1}{E_{\mathrm{th}}} \exp \left(-\frac{1}{E_{\mathrm{th}}}\left[\frac{v^{2}}{2}+\frac{\sqrt{1-2 X_{1}^{2}}}{2} u^{2}-\frac{1}{3} u^{3}\right]\right) \text {. }
$$

Note that the potential well around the SFP of 1:1 parametric resonance is lowered by the amplitude $X_{1}$ of the attractor solution. When $X_{1} \rightarrow \frac{1}{\sqrt{2}}$, the potential well disappears and the system becomes globally unstable. With the time-dependent term, the distribution can be solved by perturbation. A 

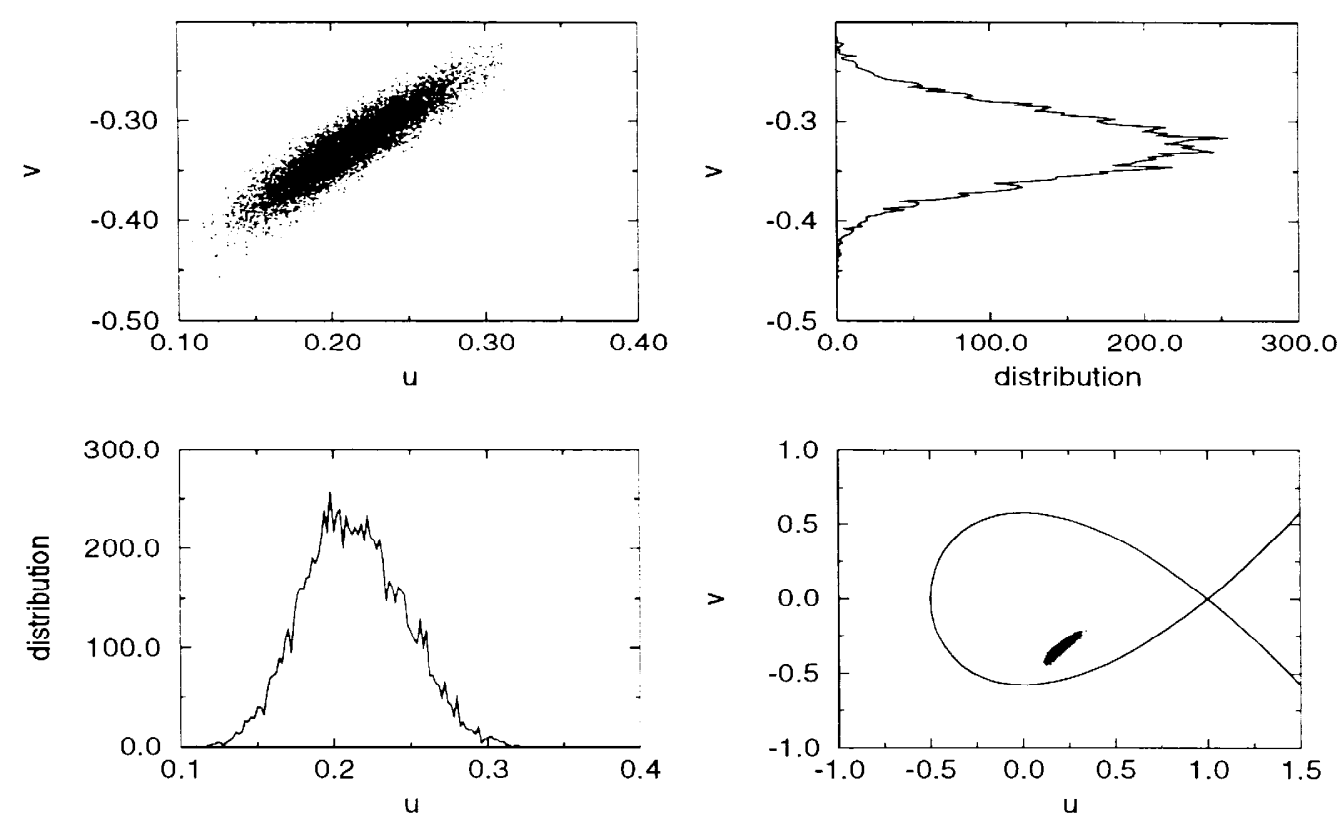

FIGURE 11. The top-left plot shows the equilibrium distribution function at a Poincaré surface of section with parameters $A=0.2, B=0.4, \omega_{m}=2.1$ and $D=0.009$. The projection of the equilibrium distribution function onto the $u$ and $v$ axes are shown respectively in the bottom-left and top-right plots. The bottom-right plot shows the relative position of the beam bunch in the QI bucket.

simulation of the steady-state distribution inside a $1: 1$ parametric resonance island is shown in Fig. 11 with $A=0.2, B=0.4, \omega_{m}=2.1$ and $D=0.009$. From the projections onto the phase and momentum axes, the rms widths $\sigma_{u}$ and $\sigma_{v}$ can be determined, and are found to be nearly equal to $D / \sqrt{2 A}$ predicted by the solution of Fokker-Planck equation.

\section{B Stability Limits}

In the presence of white noise, we expect that the resonance islands will be smeared and the global chaos will be enhanced. The plots of critical modulation amplitude $B_{c r}$ versus modulation frequency $\omega_{m}$ in Fig. 3 becomes Fig. 12. We see that white noise effectively smooths out the cusp near $\omega_{m} \approx 2$ as a result of the $1: 1$ and $2: 1$ parametric resonances. Furthermore, the quantum fluctuation can effectively reduce the parametric space where the period-two bifurcation occurs. In other words, quantum fluctuation enhances the onset of global chaos. 


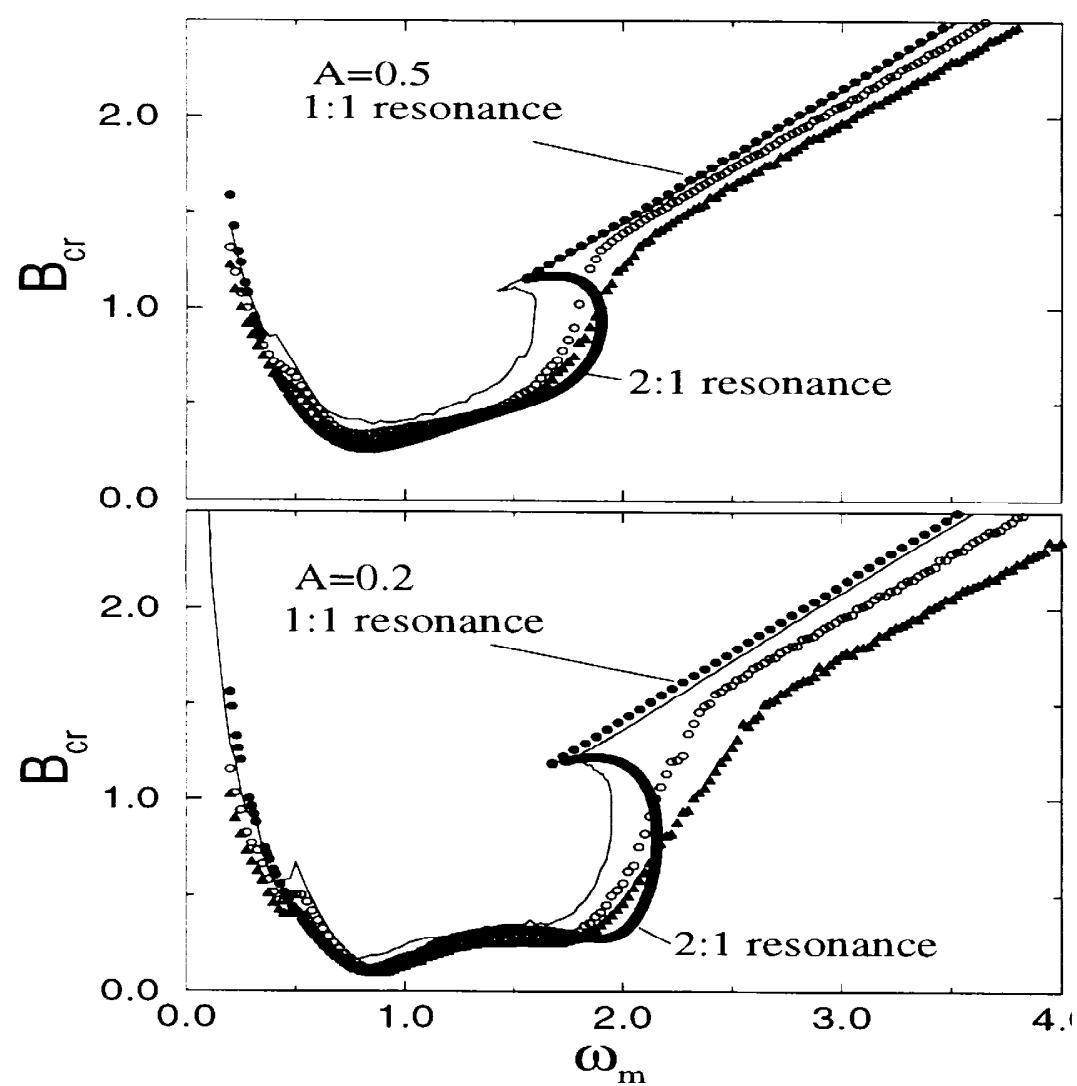

FIGURE 12. The critical phase modulation amplitude $B_{c r}$ (thin solid lines) obtained from numerical simulations is shown as function of $\omega_{m}$ for $A=0.2$ (bottom plot) and $A=0.5$ (top plot). Circle dots are $B_{c r, 1: 1}$ and $B_{2: 1}$ for the 1:1 and 2:1 parametric resonances. Note that the cusp in $B_{c r}$ obtained from numerical simulations is due to the transition from the $2: 1$ to the 1:1 parametric resonances. Including the quantum diffusion, the critical phase modulation amplitude $B_{c r}$ is reduced, where $B_{c r}$ for $D=0.03$ (open circles) and $D=0.05$ (solid triangles) are obtained by numerically integrating the stochastic differential equation.

\section{High-Frequency Modulation and Enchancement of Quantum Diffusion}

For the Mathieu-Langevin equation (5.2), which describes the motion of the quantum fluctuation $u$ about the attractor solution, we can write an equivalent Hamiltonian around the SFP:

$$
H_{1: 1}=\frac{1}{2} u^{\prime 2}+\frac{1}{2}\left[\sqrt{1-2 X_{1}^{2}}-2 X_{1} \cos \left(\omega_{m} t+\alpha\right)\right] u^{2}-\frac{1}{3} u^{3} .
$$

Therefore, with phase modulation, we have an equivalent potential well around the SFP of the 1:1 parametric resonance,

$$
V(u)=\frac{1}{2}\left(\sqrt{1-2 X_{1}^{2}}-2 X_{1} \cos \left(\omega_{m} t+\alpha\right)\right) u^{2}-\frac{1}{3} u^{3} .
$$


For very high modulation tune $\omega_{m} \gg 2$, the time-varying part can be averaged to obtain the effective potential [20],

$$
V_{\mathrm{eff}}(u)=\frac{1}{2}\left(\sqrt{1-2 X_{1}^{2}}+\frac{2 X_{1}^{2}}{\omega_{m}^{2}-\sqrt{1-2 X_{1}^{2}}}\right) u^{2}-\frac{1}{3} u^{3}
$$

The potential difference between UFP and SFP becomes

$$
\Delta V_{\mathrm{sep}}=\frac{1}{6}\left(\sqrt{1-2 X_{1}^{2}}+\frac{2 X_{1}^{2}}{\omega_{m}^{2}-\sqrt{1-2 X_{1}^{2}}}\right)^{3}
$$

Thus, there is a reduction in depth of the potential well, implying that larger quantum diffusion leads to easier particle loss. When $\Delta V_{\text {sep }}$ is substituted for $E_{1}$ in BHL lifetime formula, the enhanced loss rates shown in Fig. 13 agree with simulations.

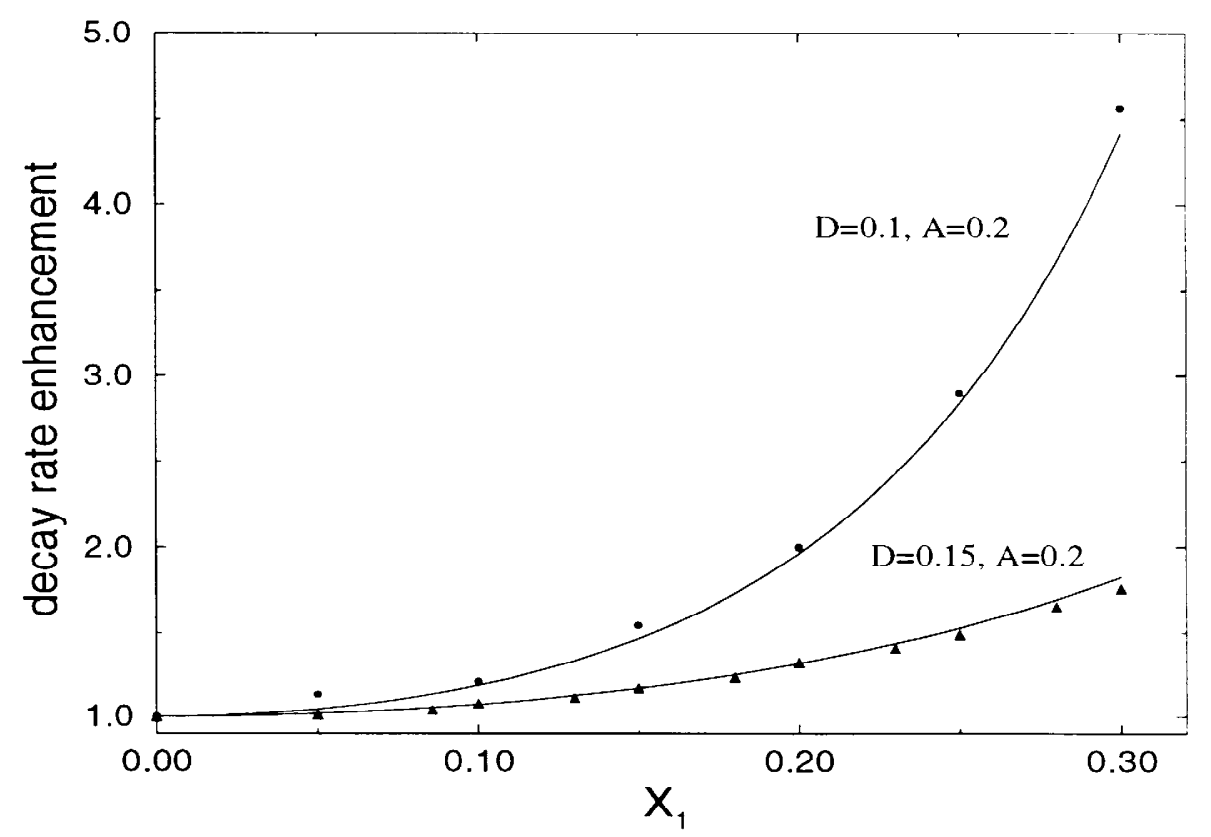

FIGURE 13. The triangles and dots show the escape rate enhancement as a function of the attractor amplitude $X_{1}$ for $A=0.2, D=0.1$ and $D=0.15$. The solid lines are obtained from the BHL formula with $E_{1}$ replaced by the separatrix energy $\Delta V_{\text {sep }}$ of Eq. (5.9). The parameter $B$ is varied to obtain appropriate $X_{1}$ for a given modulation tune $\omega_{m}=4$.

\section{Low-Frequency Modulation and Stochastic Resonances}

For low-frequency modulations, the time dependent term in the potential well of Eq. (5.7) cannot be averaged. Thus the depth of the well, or the potential difference between UFP and SFP, 


$$
\Delta V_{\mathrm{sep}}^{\mathrm{sr}}=\frac{1}{6}\left(\sqrt{1-2 X_{1}^{2}}-2 X_{1} \cos \left(\omega_{m} t+\alpha\right)\right)^{3},
$$

oscillates according to the modulation frequency and so there will be the beam loss. The upper plot of Fig. 14 shows the particle escape rate obtained from a numerical simulation as a function of time with parameters $2 \pi \nu_{s}=0.04$, $A=0.1, B=0.5, \nu_{m}=0.2$ and $D=0.075$. Note here that there are major peaks separated by $\Delta N=1 /\left(\nu_{m} \nu_{s}\right)$ turns. The solid line in the top plot shows the time dependent decay rate of the BHL formula with $E_{1}$ replaced by $\Delta V_{\text {sep }}$ of Eq. (5.10). The lower plot of Fig. 14 shows the phase of the centroid of the beam bunch as a function of time. It is clear that when the centroid of the beam bunch is near the UFP, particle loss is greatly enhanced. The occurrence of the periodic beam loss can therefore be associated with the enhancement due to the 1:1 parametric resonance. This periodic enhancement of diffusion is called the stochastic resonance. Occasionally, this type of enhancement may occur in a stochastic dynamical system with an algorithm of white noise that is not sufficiently random.

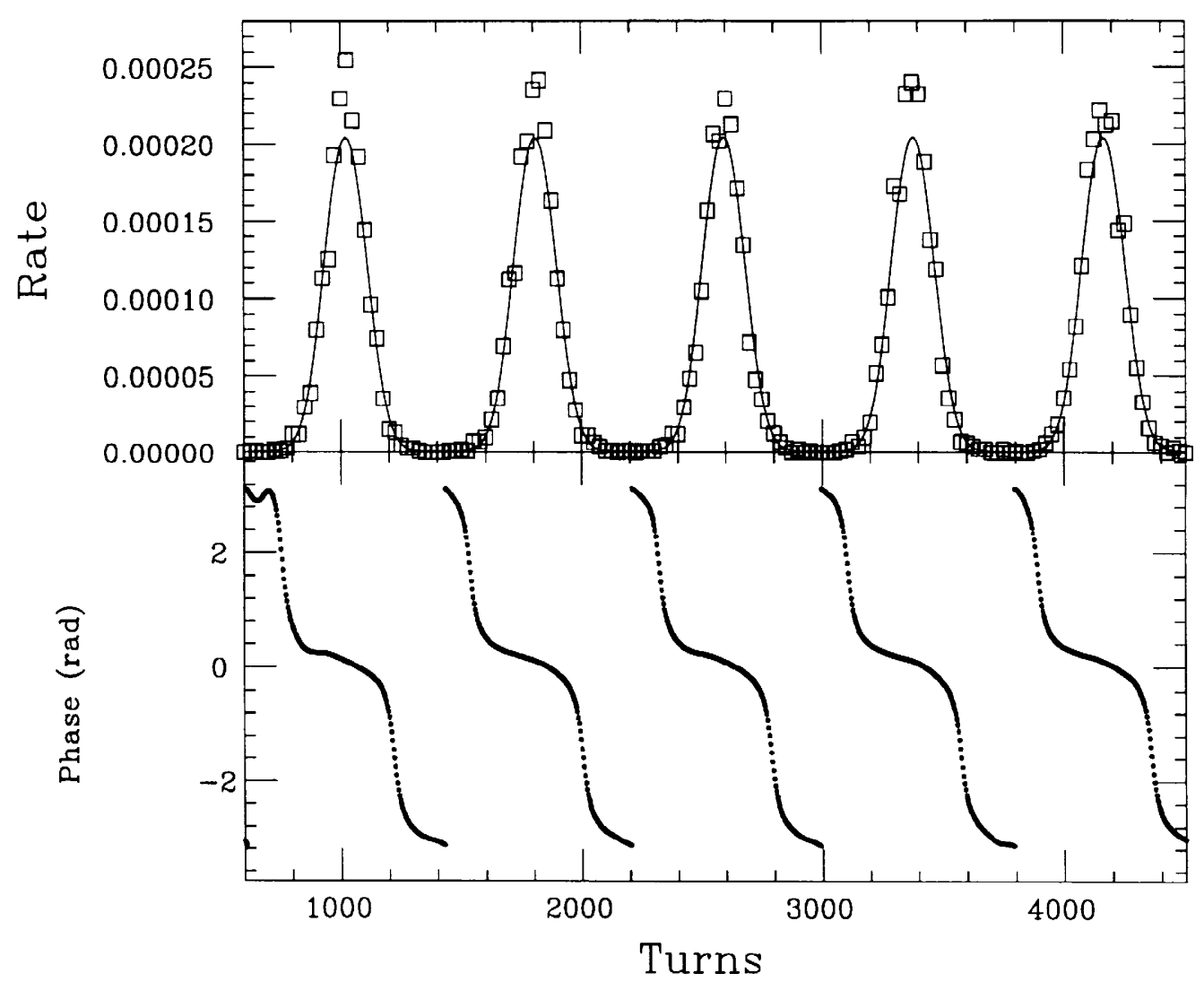

FIGURE 14. Particle escape rate $\left(1 / \tau_{q}\right)$ is plotted (upper) versus the turn numbers for $2 \pi \nu_{s}=0.04, A=0.1, B=0.5, \omega_{m}=0.2$ and $D=0.075$, to be compared with the BHL formula shown in solid. The phase of the bunch centroid is shown in the lower plot. 


\section{TOUSCHEK LIFETIME}

In rest frame of the synchronous particle of the bunch, the longitudinal momentum spread of an off-momentum particle is reduced by the Lorentz relativistic factor $\gamma$,

$$
\delta_{c}=\frac{1}{\gamma} \delta_{\mathrm{lab}}
$$

Here, both $\delta_{c}$ and $\delta_{\text {lab }}$ are measured with respect to the synchronous momentum $p_{0}$ in the laboratory frame. Due to radiation damping and quantum excitations, we have

$$
\left\langle\left(x_{\beta}^{\prime}\right)^{2}\right\rangle^{1 / 2} \gg\left\langle\left(z_{B}^{\prime}\right)^{2}\right\rangle^{1 / 2} \approx\left\langle\left(\delta_{c}\right)^{2}\right\rangle^{1 / 2}
$$

where $x_{\beta}^{\prime}$ and $z_{\beta}^{\prime}$ are the slope of the horizontal and vertical betatron oscillations. Since the horizontal momentum spread of the beam is much larger than the longitudinal momentum spread in the rest frame of the synchronous particle, large-angle Coulomb scatterings will transfer horizontal momentum into longitudinal momentum, leading to beam loss. The limited lifetime as a result of this mechanism is called Touschek lifetime [21].

For the QI bucket, $\delta_{\text {lab }}=\left|\eta_{0} / \eta_{1}\right|$ is small, so that Touschek lifetime will be much smaller than usual. This lifetime can be derived straightforwardly from the differential cross-section of Moller scattering. The Touschek lifetime $\tau_{\mathrm{T}}$ is found to be

$$
\tau_{\mathrm{T}}^{-1}=\frac{1}{N} \frac{d N}{d t}=\frac{N r_{0}^{2} c}{8 \gamma^{2} \pi \sigma_{x} \sigma_{z} \sigma_{s}}\left|\frac{\eta_{1}}{\eta_{0}}\right|^{3} D(\xi)
$$

where

$$
D(\xi)=\sqrt{\xi}\left[-\frac{3}{2} e^{-\xi}+\frac{\xi}{2} \int_{\xi}^{\infty} \frac{\ln u}{u} e^{-u} d u+\frac{1}{2}(2+3 \xi-\xi \ln \xi) \int_{\xi}^{\infty} \frac{e^{-u}}{u} d u\right],
$$

with

$$
\xi=\left(\frac{\Delta p}{\gamma \sigma_{p}}\right)_{\mathrm{lab}}^{2}=\left|\frac{\eta_{0}}{\gamma \sigma_{p} \eta_{1}}\right|^{2}
$$

and $\sigma_{x}, \sigma_{z}$, and $\sigma_{s}$ are the rms bunch widths in the horizontal, vertical, and longitudinal directions, and $\left(\sigma_{p}\right)_{\text {lab }}$ is the rms longitudinal momentum spread, all measured in the laboratory frame. This expression is illustrated in Fig. 15, where the number of particles $N$ allowed in a bunch for a 10 hour Touschek lifetime is plotted as a function of $\left|\eta_{0} / \eta_{1}\right|$, the momentum acceptance of the QI bucket. The bunch is chosen to have a rms length of $\sigma_{s}=1$ ps and 


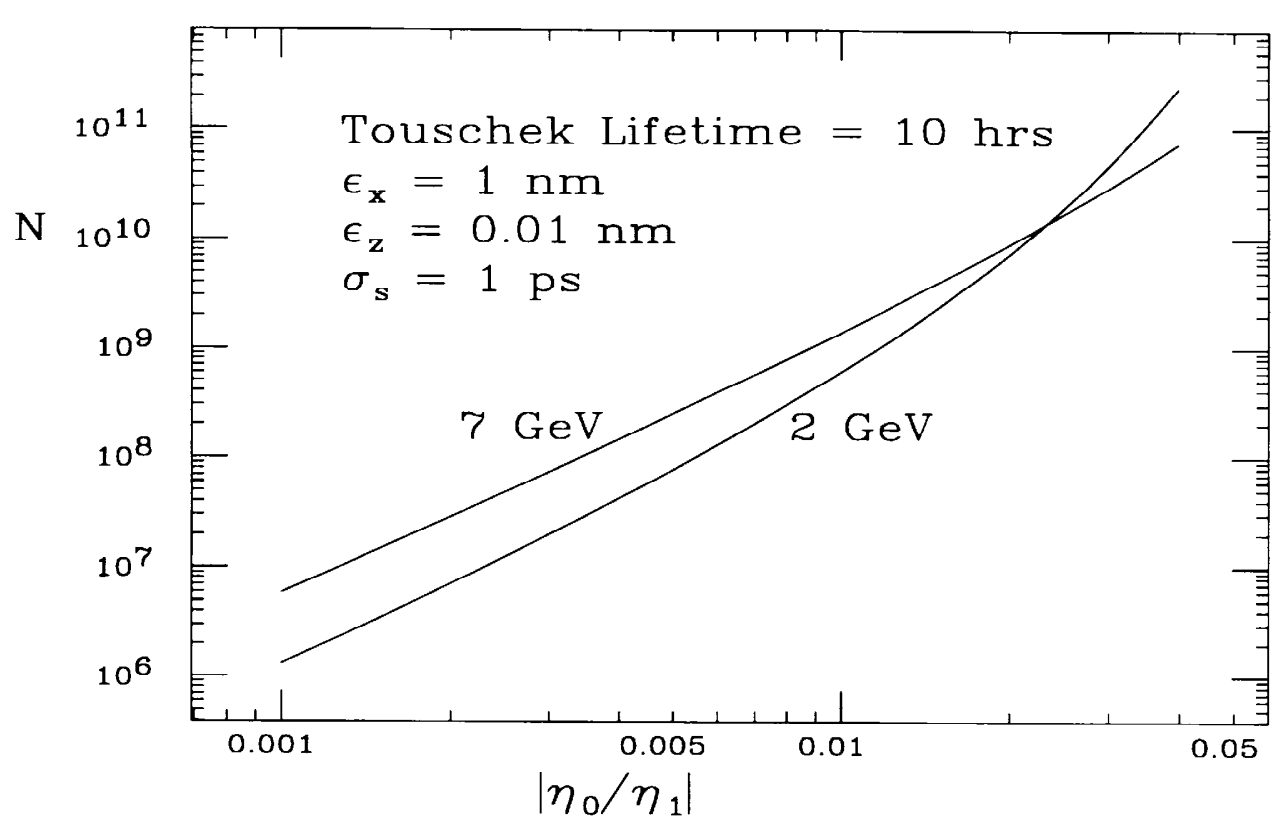

FIGURE 15. The expected number of particles per bunch in the QI storage ring limited by the Touschek lifetime of $10 \mathrm{~h}$ for electron beam energy of $2 \mathrm{GeV}$ and $7 \mathrm{GeV}$ respectively.

unnormalized rms transverse emittances of $\epsilon_{x}=1 \mathrm{~nm}, \epsilon_{z}=0.01 \mathrm{~nm}$ assuming a beta function of $10 \mathrm{~m}$. For example, at $7 \mathrm{GeV}$, with $\left|\eta_{0} / \eta_{1}\right|=0.005$, such a bunch can accommodate only $\sim 6 \times 10^{6}$ particles. To accommodate a higher intensity beam, $\eta_{1}$ must be reduced. For other bunch parameters, the tolerable beam intensity can be scaled from Eq. (6.3).

\section{CONCLUSIONS}

We have studied the kinematics of the $\alpha$-like QI bucket and found that the synchrotron frequency drops to zero at the edge of the bucket very abruptly. As a result, parametric resonances induced by time-dependent perturbations will overlap one another and give rise to chaos. In fact, the stability of the bucket is strongly affected by harmonic phase modulation. Because the QI bucket does not possess symmetry between positive and negative momentum spreads, the 2:1 parametric resonance is possible. We have shown that global chaos in this system is a result of a sequence of period-two bifurcations into 2:1 parametric resonances.

Both the normalized radiation damping parameter $A$ and quantum diffusion parameter $D$ are enhanced in the normalized equation of motion for the beam particles. Although the approach to chaos becomes worsened, the particle distribution in the longitudinal phase space turns out to remain Gaussian 
in the phase direction but non-Gaussian in the momentum direction unless when the size of the bunch is small. The rms spreads in both projections are governed by the "thermal energy" $E_{t h}=D^{2} /(2 A)$ according to Einstein's relation. In spite of enhanced damping and diffusion, the quantum lifetime of a bunch in the QI bucket is longer than expected. Phase modulation will enhance diffusion and thus shorten the quantum lifetime if the modulation frequency is high. At low modulation frequency, however, particle loss oscillates at the modulation frequency whenever the bunch is near the unstable fixed point, producing a phenomenon called stochastic resonance. Because of the small momentum acceptance of the $\alpha$-like QI bucket, particle loss due to Touschek scattering becomes more severe. The tolerable bunch intensity for a fixed Touschek lifetime has been computed.

Voltage modulation can also affect the stability of the QI bucket. However, its effect is much smaller than that of the phase modulation, and therefore the discussion has been omitted. It is also interesting to point out that the potential-well distortion of the bunch due to the coupling impedance of the vacuum chamber will not be affected by the enhancement of the damping parameter and diffusion parameter.

It appears that the $\alpha$-like QI bucket may become indispensable in producing ultra short bunches. Therefore, its detailed study has become rather important. Experiments should be performed to confirm some of the predictions given in this presentation.

\section{REFERENCES}

1. K.R. Symon and A.M. Sessler, Proc. 1956 Int. Conf. on IIigh Energy Accelerators, Geneva, 1956, p. 44.

2. C. Pelligrini and D. Robin, Nucl. Inst. Methods, A 301, 27 (1991).

3. D. Robin, E. Forest, C. Pelligrini, A. Amiry Phys. Rev. E 48, 2149 (1993). H. Bruck et al., IEEE Trans. on Nucl. Science NS20, 822 (1973).

4. L. Liu et al., Nucl. Inst. and Methods A329, 9 (1993).

5. H. Hama, S. Takano and B. Isoyoma, Nucl Inst. and Methods A329, 29 (1993). S. Takano, H. Hama and G. Isoyama, Japan J. of Appl. Phys. 32, 1285 (1993).

6. A. Nadji et al., Proceedings of the 4th European particle accelerator conference, p. 128 (1994).

7. D. Robin, H. Hama, and A. Nadji, Lawrence Berkeley Tech-note LBL-37758 (1995).

8. D. Robin et al., SLAC-PUB-95-7015 (1995)

9. A. Riabko et al., Particle dynamics in quasi-isochronous storage rings, Phys. Rev. E54, 815 (1996). (1996).

10. D. Jeon, etal., Role of parametric resonances in global chaos, accepted for publication in Phys. Rev. E.

11. M. Bai, etal., Stochastic beam dynamics in quasi-isochronous storage rings, accepted for publication in Phys. Rev. E. 
12. Handbook of Mathematical Functions, edited by M. Abramowitz and I.A. Stegun, (National Bureau of Standards, 1975).

13. I.S. Gradshteyn and I.M. Ryzlik, Table of Integrals, Series, and Products, (Academic Press, New York, 1980).

14. S.Y. Lee et al., Phys. Rev. E 49, 5717 (1994); J.Y. Liu et al., Phys. Rev. E 50, R3349 (1994). J.Y. Liu et al., Particle Accelerators, 49, 221 (1995).

15. D. Li, et al., Phys. Rev. E48, R1638 (1993); D. Li, ct al., Nucl. Inst. and Methods A 364, 205 (1995).

16. H. Huang, et al.. Phys. Rev. E48, 4678 (1993); M. Ellison, et al., Phys. Rev. Lett. 70, 591 (1993); M. Syphers, et al., Phys. Rev. Lett.. 71, 719 (1993); Y. Wang, et al., Phys. Rev. E 49, 1610 (1994).

17. H.A. Kramers, Physica (Utrecht) 7, 284 (1940).

18. S. Chandrasckhar, Rev. of Modern Physics 15, 1 (1943).

19. M. Büttiker, E.P. Harris, and R. Landerauer, Phys. Rev. B28, 1268 (1983).

20. L.D. Landau and E.M. Lifshitz, Mechanics, 2nd ed. (Oxford. New York, Pergamon Press, 1969).

21. C. Bernardini et al., Phys. Rev. Lett. 10, 407 (1963). 